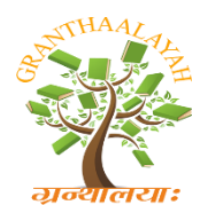

INTERNATIONAL JOURNAL OF RESEARCH GRANTHAALAYAH A knowledge Repository

Science

\title{
A SHORT REVIEW: PRODUCTION AND CHARACTERIZATION OF WINE FROM FIG FRUIT (FICUS CARICA)
}

\author{
Annavarapu Venkata Naga Kaumudi Prabha ${ }^{1}$ \\ ${ }^{1}$ Department of Food Technology, SET, Jain University, India
}

\begin{abstract}
Fig is one of the oldest fruit species cultivated in the Mediterranean basin, and it can be widespread in warm and dry climates around the world. Turkey is the primary producer and the tree can bear up to two crops per year which is depending upon the fig type, quality can be differing from season to season. Figs can be eaten fresh, dried, or processed into different types of products. They are low in organic acids and high in sugars so it is sweet-tasting fruit. Their phenolic content is medium and higher in red cultivars which hold high levels of anthocyanins. The total carotenoid in the fig is quite low and mostly concentrated in the fruit's peel when compared with other fruits. Fruit drying is the easiest way of fig fruit preservation. The traditional sun-drying method yields produce with diverse quality. Automated air dehydration has several advantages and makes fruit with high sugar content and high total phenolics. Anthocyanins and carotenoids are lost during the drying process. The consumption of figs should be encouraged as it contains high beneficial effects and potential healthy alternative for sweets.
\end{abstract}

Keywords: Organic Acids; Phenolics; Sugars; Carotenoids; Fig; Dried; Fresh.

Cite This Article: Annavarapu Venkata Naga Kaumudi Prabha. (2018). "A SHORT REVIEW: PRODUCTION AND CHARACTERIZATION OF WINE FROM FIG FRUIT (FICUS CARICA)." International Journal of Research - Granthaalayah, 6(3), 134-139. https://doi.org/10.29121/granthaalayah.v6.i3.2018.1506.

\section{Introduction}

Ficus (Moraceae) is one of the largest genera with more than 800 species of trees, shrubs, climbers, and creepers in the tropics and subtropics worldwide. This genus is an important genetic resource due to its high economic and nutritional properties and also an important part of the biodiversity. It is also a good source of food for fruit-eating animals. 23 species of hemiepiphytes and lithophytes are present which produce aerial and creeping root systems.

F. carica L. is an important member of the genus Ficus. It is ordinarily deciduous (leaves shedding annually) and commonly referred to as "fig". It is the first plant cultivated by humans and the tree native is southwest asia and the eastern Mediterranean.. Its common edible part is 
the fruit which is fleshy, hollow, and receptacle. The dried fruits of figs contain important source of vitamins, minerals, fibers, polyphenols, carbohydrates, sugars, organic acids, and phenolic compounds. It holds an excellent source of phenolic compounds, such as proanthocyanidins. Fruit, root, and leaves of these plants are used in traditional medicine to treat various diseases such as gastrointestinal, respiratory, and cardiovascular disorders. F. carica L. belongs to the order of Urticales and family of Moraceae with over 1400 species classified into about 40 genera A number of these plants are female and produce only a seed-bearing fruit, whereas others are male and produce only pollen and pollen-carrying wasp progeny. The barks are grayish and slightly roughened. The leaves are stipulated and with obovate, nearly leaf blade, palmately lobed, cordate base, undulate or irregularly dentate margin, acute to obtuse apex, and scabrous-pubescent surfaces. . Turkey, Egypt, Morocco, Spain, Greece, Brazil, and other places with typically winters and hot summers are the major producers of edible figs Fruits can be eaten raw, dried, canned, or in other preserved forms Morphological data explains that the fig is gynodioecious, whereas from a functional point, the fig is considered dioecious with two tree morphs: Capri fig and edible fig. Cultivation of habitual fig areas have been decreased and genetic variability was reduced due to disappearance of many cultivars. It can only be pollinated by their associated agaonidwasps, and in turn the wasps can only lay eggs within their specific fruit. For pollination and reproduction of these species of to occur, its associated pollinator wasp must be present. Conversely, for reproduction of agaonid wasps to occur, their associated species of Fig must be present. Blastophagapsenes (L.) is the pollinator wasp for F.Carica.

\subsection{Phytochemistry}

F. carica contains several bioactive compounds such as phenolic compounds, phytosterols, organic acids, anthocyanin composition, triterpenoids, coumarins, and volatile compounds such as hydrocarbons, aliphatic alcohols, and few other compounds of secondary metabolites from different parts of F. carica . Most species of F. carica contain phenolic compounds, organic acids, and volatile compounds.

The leaves of F. carica contains large number of volatile compounds which are identified and distributed by distinct chemical classes, such as aldehydes: methyl-butanal, (E)-2-pentanal, hexanal, and (E)-2-hexanal,2-methylbutanal alcohols: 1-penten-3-ol, 3-methyl-1-butanol, 2methylbutanol, heptanol, benzyl alcohol, (E)-2-nonen-1-ol, and phenylethyl alcohol, ketone: 3pentanone, esters: methyl butanoate, methyl hexanoate, hexyl acetate, ethyl benzoate, and methyl salicylate, monoterpenes: limonene and menthol, sesquiterpenes: -cubenene, -guaiene, -ylangene, copaene, -bourbonene, -elemene, -gurjunene, -caryophyllene, -cubebene, aromadendrene, caryophyllene, -muurolene, -cadinene, -muurolene, germacrene $\mathrm{D}, \quad$ and (+)-ledene, norisoprenoid: -cyclocitral, and Miscellaneous compounds: psoralen fifteen anthocyanin pigments were isolated from the fig fruit and bark of F. carica. Most of them contain cyanidin as aglycone and some pelargonidin derivatives Total and individual phenolic compounds, phenolic acid, chlorogenic acid, flavones, and flavonols, have been isolated from fresh and dried fig skins of F. carica and dried figs contain higher amounts of phenolics than the pulp of fresh fruits. Quercetin rutinoside is one of the major phenolic compounds Phenolic acids; 3-O- and 5-Ocaffeoylquinic acids, ferulic acid, quercetin-3-O-glucoside, quercetin-3-O-rutinoside, psoralen, and bergapten, and organic acids (oxalic, citric, malic, shikimic, and fumaric acids) were isolated 
from the pulps and peels of figs Phenolics, anthocyanins, fructose, glucose, and sucrose were identified from the fig of F. carica

\subsection{Wine Preparation}

In the preparation of figwine Microorganism and inoculums were prepared .Preperation includes: S. cerevisiae var. ellipsoid us CFTRI 101 (wine yeast) . Culture maintenance and inoculums preparation was done. The culture was maintained on MPYD (Malt extract $0.3 \%$, Peptone $0.5 \%$, Yeast extract $0.3 \%$, Dextrose $2 \%$ and Agar $1.5 \%$ ) slants at $4{ }^{\circ} \mathrm{C}$. The inoculum was prepared by inoculating the slant culture into $25 \mathrm{ml}$ of the sterile MPYD liquid medium taken in $100 \mathrm{ml}$ Erlen Mayer flask and allowed to grow it on a rotary shaker $(100 \mathrm{rpm})$ for $48 \mathrm{~h}$ at $37^{\circ} \mathrm{C}$. This inoculum $(3 \times 106$ cells $/ \mathrm{ml})$ was transferred to 250 mlconical flasks having $100 \mathrm{ml}$ fig juice. For the evaluation of yeast strains on fermentation three types of yeast strains including S. cerevisiae var. ellipsoid us CFTRI 101, Palm Wine Isolate (PWY1) and Baker's Yeast were taken up.

\subsection{Fermentation}

Batch fermentation of the inoculated should carry out in a conical flasks by incubating at $\mathrm{pH} 4.5$ and at temperature $20^{\circ} \mathrm{C}$ for 15 days at $22 \pm 20 \mathrm{C}$. The samples were collected by separation of the cells by centrifugation at $5,000 \times \mathrm{g}$ for 10 minutes. Chemical and sensory analyses were evaluated by keeping the fermented samples at $-20^{\circ} \mathrm{C}$ for a few weeks Effect of enzyme treatment, effect of pectinase enzyme treatment and fermentation by yeast cultures using three different yeast strains on ethanol production was identified. The pectinase enzyme concentration used was $0.6 \%$ and fermentation was conducted at $30 \mathrm{o} \mathrm{C}$ at $\mathrm{pH} 4.5$ in fig must for a period of 12 to 120 hours. Observations were taken at an interval of 12 hours. Composition of volatiles from three fig cultivarsBlack Mission, Calimyrna, and Kadotafermented at 25 $\pm 2 \mathrm{o} \mathrm{C}$ and $\mathrm{pH} 4.5$ for 10 days was also studied. 'Effect of different conditions on the storage of wine fig wine was packed in bottles of different colours, viz. brown, green, white and was stored at a temperature of 6, 16 and $260 \mathrm{C}$. The changes in optical density at $420 \mathrm{~nm}$ were observed.

\subsection{Physico-Chemical, Micro-Biological and Sensory Analysis}

Shaffer \& Somogyi11 method is used for the concentration of reducing sugars. Total dissolved solids were measured by estimating specific gravity of water soluble portion of the juice obtained by the centrifugation at $10,000 \times \mathrm{g}$ for $15 \mathrm{~min}$. Densitometer is used to determine the specific gravity at $20^{\circ} \mathrm{C}$. The results are converted to grams of dissolved solids per $100 \mathrm{ml}$ and expressed as grams of sucrose. Total acidity was determined by titration with $0.1 \mathrm{~N} \mathrm{NaOH}$ expressed in tartaric acid and volatile acidity is expressed in acetic acid $\mathrm{mg} / 100 \mathrm{ml}$. Phenols of the fig juice and wines were estimated by Folin-Ciocalteau method12. Effect of storage, colour stability of stored wines was measured as wine colour OD at $420 \mathrm{~nm}$. Ethanol and other metabolites (glycerol, methanol and total esters) were determined with the help of Gas Chromatography 13,14 . The fermented samples were centrifuged at $5000 \times \mathrm{g}$ for $10 \mathrm{~min}$. Ethanol analysis is used for the supernatant .Nitrogen was used as carrier gas and $2 \mathrm{~m}, 2 \mathrm{~mm}$ ID1/4 mm quantity with a flow of $20 \mathrm{ml} / \mathrm{min}$ was applied. The eluted compounds were identified by flame ionization detector (FID), for this the fuel gas used was hydrogen with a flow rate of $40 \mathrm{ml} / \mathrm{min}$. and the oxidant was air with a flow rate of $40 \mathrm{ml} / \mathrm{min}$. and n-propanol was used as internal 
standard. The analysis of total volatile compound composition was carried out on a HewlettPackard series 6890 gas chromatograph linked to an HP-5973 mass-selective detector, with a $30 \mathrm{~m} \times 0.25 \mathrm{~mm}$ i.d., $0.25 \mu \mathrm{m}$ film thickness HP-5MS (Agilent, Palo Alto, CA fused silica capillary column All the experiments were carried out three times (triplicate) and the mean value of the these three experiments are presented. Sensory evaluation of wine samples were performed by 6 well trained panellists. Different attributes of produced fig wine included were: aroma, visual, harmony and taste . The system contains total 20 points including, 2 points for colour, 2 points for clarity, 4 points for smell and 12 points for taste 15 .

\section{Results and Discussions}

Screening of fig cultivars the fruits of different cultivars differ in sugar concentrations and other physico-chemical characteristics. 'Black mission gave highest juice yield followed by calimyrna and least by kodota'. The main important character of juice for fermentation is sugar content. The total soluble solids (TSS) of the mango must range from 22.7 and 27.2 per cent. The high TSS was from 'black mission' (20.5\%) followed by 'calimyrna and kodota (20.1 and 16.5\%, respectively). The sugar content of must range from 15 to $18 \%(\mathrm{w} / \mathrm{v})$ while titrable acidity as tartaric acid range from 0.310 to $0.462 \%(\mathrm{w} / \mathrm{v})$. The $\mathrm{pH}$ of the juice musts was 4.8 . Higher alcohols may influence sensory characteristics although they are relatively lesser quantity of the total substances. Fermentation changed the aroma of fruit juice, because of the presence of yeast volatiles and the metabolism of original fruit volatiles. The concentration of esters in wine varied from 15 to $35 \mathrm{mg} / \mathrm{l}$ and it was affected by many factors like variety of fruit, clarification and fermentation conditions. The concentration of total polyphenols was different in wine from cultivar to cultivar of fresh figs.. In all the cases the phenolic concentration increased after the fermentation. Phenolic compounds are most important compounds in determining the quality of the wine, because they greatly influence colour, bitterness, astringency and chemical stability of the wine.

Effect of pectinase on juice yield and ethanol production Pectinase treatment increased the yield of juice in all fig cultivars. The proper pectinase enzyme concentration was $0.6 \%$ and treatment time was oduced from 'black mission followed 12 hours. It was considered very important because higher concentration may result in increased methanol production due to the hydrolysis of methyl pectin by methyl pectin esterases (unpublished data). Pectinase treatment probably increased the ethanol production from fig pulp by all three yeast strains. The increase in ethanol may be because of clarity and increased sugar concentration in pectinase treated samples it is observed as increase in iso-amyl alcohol and 2-phenyl ethanol and a decrease in n-propanol concentrations in fermented macerated grapes pre-treated with pectolytic enzymes.

\subsection{Effect of Yeast Strains on Wine Production}

To evaluate the fermentation performance, the yeast strains were kept in different temperatures and $\mathrm{pH}$ during fermentation. Out of the three yeast strains S. cerevisiae CFTRI 101 gives best results The yeast strain isolated from palm wine (PWY1) will show better results than the commercial Baker's yeast The Baker's yeast could not ferment proper ethanol production, The CFTRI 101 strain showed high fermentation during wine production and it suits to all the conditions and started ethanol production within $4 \mathrm{~h}$ of inoculation and completed the 
fermentation within $70-76 \mathrm{~h}$ and it showed good ethanol tolerance capacity up to $10 \%(\mathrm{w} / \mathrm{v})$. The higher alcohol concentration differs in wines produced by three yeast strains. The strain CFTRI 101 produces higher concentrations of alcohols compared to the other two strains that ranged between 150-300 mg/l by CFTRI 101 followed by Baker's yeast 123- $200 \mathrm{mg} / \mathrm{l}$ and PWY1 100$185 \mathrm{mg} / 1$. . Ethyl acetate, along with the total esters, is helpful to the aroma of wine.. The greatest synthesis took place during the fermentation at 50-75\% of the sugars, more as in the samples inoculated with CFTRI 101 yeast strain, which shows a greater capacity for generating these volatile compounds than the other two yeast strains. The levels of higher alcohols in wines produced from same variety of fruits will differ with yeast used for the fermentation and the yeast cell plays an important role in formation of fuel oils of higher alcohols. The amount of ester production is different with the three yeasts. These esters at higher concentrations, which are above a threshold value of $150 \mathrm{mg} / \mathrm{l}$, would give vinegar smell to the wine. In the present study, none of the strain produced high quantities of these esters in wine. The formation and concentration of the volatile compounds found in fig wine were dependent on the fig cultivar and S. cerevisiae strain used.

\section{Conclusions}

It can be concluded that 'black mission, calymirna and kodotacultivars are most suitable for fig wine production. The sensory evaluation has indicated that the wine possesses novel characteristics in aroma and taste and good acceptability. 'Blackmissionwine fermented with yeast strain CFTRI 101 was most acceptable followed by 'calymirna and kodota. Information on an adequate process for both juice and wine production from figs and other tropical fruits and the findings regarding pectinase treatment, formation of major volatile compounds in wine industry are noted.

\section{Acknowledgements}

We thank the DST, FIST, No. SR/FST/ETI-378/2014, Government of India for the award of research project and Ministry of Food Processing Industries (MOPFPI) for the support to complete this project.

\section{References}

[1] Al-Kahtani, H. A., Abu-Tarboush, H. M., Bajaber, A. S., Atia, M., Abou-Arab, A. A., \& El Mojaddidi, M. A. (1996). Chemical changes after irradiation and post-irradiation in tilapia and Spanish mackerel. Journal of Food Science, 61(4), 729-733.

[2] Brannan, R. G. (2008). Effect of grape seed extract on physicochemical properties of ground, salted, chicken thigh meat during refrigerated storage at different relative humidity levels. Journal of Food Science, 73(1), C36-C40.

[3] Brannan, R. G. (2009). Effect of grape seed extract on descriptive sensory analysis of ground chicken during refrigerated storage. Meat Science, 81(4), 589-595.

[4] Brannan, R. G., \& Mah, E. (2007). Grape seed extract inhibits lipid oxidation in muscle from different species during refrigerated and frozen storage and oxidation catalyzed by peroxinitrite and iron/ascorbate in a pyrogallol red model system. Meat Science, 77(4), 540-546.

[5] Cheynier, V., \& Rigaud, J. (1986). HPLC separation and characterization of flavonols in the skins of Vitis vinifera var. Cinsault. American Journal of Enology and Viticulture, 37 (4), 248-252. 
[6] Decker, E. A., \& Crum, A. D. (1991). Inhibition of oxidative rancidity in salted ground pork by carnosine. Journal of Food Science, 56(5), 1179-1181.

*Corresponding author.

E-mail address: kaumudiprabha1996@ gmail.com 